\title{
Insuficiência de vitamina $D$ e fatores associados: um estudo com idosos assistidos por serviços de atenção básica à saúde
}

\author{
Vitamin D insufficiency and factors associated: a study with older \\ adults people from primary health care network
}

Patricia Moreira Donato Rolizola (https://orcid.org/0000-0001-9463-8686) ${ }^{1}$ Carolina Neves Freiria (https://orcid.org/0000-0002-1493-3202) ${ }^{2}$

Graziele Maria da Silva (https://orcid.org/0000-0003-0634-275X) ${ }^{1}$

Tabatta Renata Pereira de Brito (https://orcid.org/0000-0001-9466-2993) ${ }^{3}$

Flávia Silva Arbex Borim (https://orcid.org/0000-0001-7316-1145) ${ }^{4}$

Ligiana Pires Corona (https://orcid.org/0000-0001-5298-7714) ${ }^{1}$
${ }^{1}$ Faculdade de Ciências Aplicadas, Universidade Estadual de Campinas. R. Pedro Zaccaria 1.300 . 13484-350 Limeira SP Brasil.

pmdonator@gmail.com ${ }^{2}$ Faculdade de Ciências Médicas, Universidade Estadual de Campinas. Campinas SP Brasil. ${ }^{3}$ Faculdade de Nutrição, Universidade Federal de Alfenas. Alfenas MG Brasil. ${ }^{4}$ Departamento de Saúde Coletiva, Faculdade de Ciências da Saúde, Universidade de Brasília. Brasília DF Brasil.

\begin{abstract}
This article aims to analyze vitamin $D$ insufficiency and factors associated among older adults using primary health care services. This is a cross-sectional study that evaluated 533 older adults individuals ( $\geq 60$ years old) in three cities in the state of São Paulo, Brazil. Serum level of 25-hydroxyvitamin D (25-OHD) was evaluated by chemiluminescence. The factors evaluated were sociodemographic information (sex, age group, ethnicity, education, income, marital status), health conditions (reported diseases), body composition (BMI, waist circumference), lifestyle (physical activity and smoking), and sun exposure (purpose, duration, frequency, time of exposure, exposed body parts, use of sunscreen, skin type). The prevalence of vitamin $D$ insufficiency was $64.5 \%$, presenting association with female participants, non-white/unreported ethnicity, low weight, high waist circumference (risk for CVD - cardiovascular disease), and physical inactivity. Negative association was observed with habitual sun exposure of hands, arms and legs, during leisure activities, daily commuting and physical activity, and between 9 am and $3 \mathrm{pm}$. The findings show the relevance of factors such as sex, ethnicity, body composition, physical activity, and sun exposure habits in the high prevalence of inadequate levels of vitamin D among older adults.
\end{abstract}

Key words Elder people, Vitamin D, Sun exposure, Public health.
Resumo O objetivo deste artigo é analisar a insuficiência de vitamina $D$ e fatores associados em idosos assistidos na atenção primária à saúde. Trata-se de um estudo transversal que avaliou 533 idosos ( $\geq 60$ anos) em três cidades do estado de São Paulo, Brasil. Foi avaliada a 25-hidroxivitamina D (25 OH D) sérica por quimioluminescência. Os fatores avaliados foram condições sociodemográficas (sexo, faixa etária, etnia, escolaridade, renda, estado civil), de saúde (doenças referidas), composição corporal (IMC, circunferência da cintura), estilo de vida (atividade física e tabagismo) e exposição solar (finalidade, duração, frequência e horário de exposição, partes expostas, uso de protetor solar, tipo de pele). A prevalência de insuficiência foi de $64,5 \%$, com associação para sexo feminino, etnia não brancos/não declarados, baixo peso, circunferência da cintura elevada (risco para DCV) e inatividade física. Houve associação negativa para exposição solar habitual de mãos, braços e pernas, durante atividade de lazer, deslocamentos diários e atividade física e entre as $9 \mathrm{he}$ 15h. Os achados mostram a relevância de fatores como sexo, etnia, composição corporal, atividade física e hábitos de exposição solar na alta prevalência de níveis inadequados de vitamina $D$ em idosos.

Palavras-chave Idosos, Vitamina D, Exposição solar, Saúde pública 


\section{Introdução}

A vitamina $\mathrm{D}$ tem seu papel muito bem reconhecido na mineralização óssea e vem se destacando por sua complexa atividade no organismo, inclusive no controle de processos metabólicos. Sugere-se que o status adequado de vitamina D contribua na proteção contra distúrbios musculoesqueléticos, doenças infecciosas, cânceres, doenças autoimunes e cardiovasculares, diabetes mellitus e disfunções neurocognitivas, sendo observado que baixas concentrações séricas da vitamina se relacionam ao risco aumentado para desenvolvimento e progressão dessas doenças ${ }^{1}$.

A síntese cutânea a partir da luz solar é a principal fonte de vitamina $\mathrm{D}$ em humanos, sendo assim, existe uma falsa impressão de que em países ensolarados a deficiência de vitamina $D$ não é um problema, e por isso muitas vezes as concentrações séricas desta vitamina na população acabam não sendo testadas. No entanto, de acordo com estudos internacionais e brasileiros, a hipovitaminose D é altamente prevalente, independentemente da região avaliada, sendo que estudos nacionais mostram prevalências que variam de $28,5 \%$, em pesquisa realizada com idosos hipertensos em João Pessoa/PB durante a primavera e verão, a quase $96 \%$, em outra que avaliou idosos em São Paulo/SP durante o inverno².

Estudo de Cabral e colaboradores ${ }^{3}$, que avaliou 234 idosos do sexo masculino em Recife/ PE durante o verão, encontrou prevalência de insuficiência de $66,7 \%$, com médias de concentração sérica de 25-hidroxivitamina D abaixo do recomendado $(27,86 \pm 13,52 \mathrm{ng} / \mathrm{mL})$, similares às encontradas em outro estudo recente ${ }^{4}$, realizado com 287 idosos de comunidade em Palhoça/SC $(26,09 \pm 9,20 \mathrm{ng} / \mathrm{mL})$.

Além da exposição solar, muitos outros fatores podem estar envolvidos na deficiência dessa vitamina, como idade, pigmentação da pele, obesidade, multimorbidade e uso de determinados medicamentos, como anti-hipertensivos, anticonvulsivantes, anti-inflamatórios e sedativos. Devido aos hábitos de estilo de vida, polifarmácia, multimorbidade e eficácia reduzida da produção de vitamina $\mathrm{D}$ na pele, os idosos constituem um dos mais importantes grupos em risco de deficiência dessa vitamina ${ }^{5}$.

A partir desse contexto, considerando que existem poucos trabalhos que avaliem amostras representativas de idosos assistidos pela atenção básica no país ${ }^{6,7}$ e sabendo que a vitamina $\mathrm{D}$ sérica não é amplamente testada nessa mesma população, há a necessidade de tentar associar fatores que possam ser utilizados para a identificação precoce da insuficiência de vitamina $\mathrm{D}$, possibilitando ações e estratégias de prevenção e controle dessa condição, que vem se mostrando cada vez mais prevalente no Brasil e no mundo. O presente estudo tem por objetivo analisar a prevalência de insuficiência de vitamina $\mathrm{D}$ e sua associação com variáveis sociodemográficas, hábitos de exposição solar, condições físicas e de saúde em idosos atendidos na atenção primária à saúde.

\section{Métodos}

\section{Participantes}

Trata-se de um estudo com delineamento transversal, parte de uma pesquisa maior, intitulada "Avaliação da prevalência de deficiência de micronutrientes em idosos residentes em cidades da região de Campinas/SP", realizada nos municípios paulistas de Limeira, Piracicaba e Campinas, Brasil.

Os critérios de inclusão para participação no estudo foram: idade de 60 anos ou superior, ser morador de um dos municípios participantes e apresentar condições neurológicas e cognitivas adequadas para responder aos questionários (percebidas pelo entrevistador durante a apresentação da pesquisa e do convite de participação). Os critérios de exclusão foram: uso de suplementos alimentares à base de vitaminas e/ou minerais; estar sob acompanhamento por programa de atenção domiciliar; estar em tratamento quimioterápico.

Foi estimada uma amostra com base no número total de habitantes com idade de 60 anos e mais dos municípios de Campinas, Limeira e Piracicaba, divulgado nas estimativas populacionais para o ano de 2018, e considerando-se uma prevalência de $60 \%$ dos idosos com deficiência de pelo menos um dos nutrientes avaliados, com erro de amostragem de $10 \%$ e nível de $95 \%$ de confiança. Com isso, a amostra prevista total foi de 600 idosos, sendo 250 participantes na cidade de Campinas, 170 em Limeira e 180 em Piracicaba.

Foram coletados dados de 612 participantes no período de outubro de 2018 a dezembro de 2019 (não houve coleta durante o inverno). Para o presente estudo, foram excluídos 17 indivíduos com dados incompletos e 62 que relataram uso de suplementos contendo vitamina $\mathrm{D}$, totalizando uma amostra final de 533 indivíduos.

O recrutamento dos idosos voluntários se deu a partir de convite realizado nas Unidades 
Básicas de Saúde (UBS) indicadas pelas respectivas Secretarias de Saúde de cada município.

A pesquisa foi aprovada pelo Comitê de Ética em Pesquisa com Seres Humanos da Universidade Estadual de Campinas, sob o parecer $n^{\circ}$ 2.878.652 e CAAE 95607018.8.0000.5404. Todos os participantes assinaram o Termo de Consentimento Livre e Esclarecido.

\section{Coleta de dados}

Em cada unidade de saúde selecionada, os idosos eram convidados, pela própria equipe, a participar da pesquisa e orientados a comparecer em jejum de pelo menos oito horas na data agendada. Nesse dia, era realizada coleta da amostra de sangue, aferição de dados antropométricos e, em seguida, conduzida uma entrevista para preenchimento do questionário. Esses procedimentos foram realizados por uma equipe de estudantes de graduação e pós-graduação da área de saúde, previamente treinados pelos coordenadores da pesquisa.

\section{Variáveis do estudo}

A variável dependente do estudo foi insuficiência de vitamina D. A análise das concentrações séricas de vitamina $\mathrm{D}$ foi realizada por meio de amostras de sangue, pela dosagem da 25-hidroxivitamina D (25 OH D), pelo método de ensaio de quimioluminescência, utilizando o kit reagente Alinity 25-OH Vitamin D (Abbott). Os tubos contendo sangue coletado foram armazenados em caixa térmica com gelo e enviados imediatamente ao final da coleta para o mesmo laboratório privado contratado para a pesquisa (Pasteur), onde as amostras foram processadas e analisadas em seguida (sem congelamento). Para as concentrações séricas de 25-hidroxivitamina D, utilizou-se os valores de referência recomendados no Consenso da Sociedade Brasileira de Endocrinologia e Metabologia (SBEM) e pela Sociedade Brasileira de Patologia Clínica/Medicina Laboratorial $(\mathrm{SBPC})^{8}$, sendo considerado insuficiência de vitamina $\mathrm{D}$ valores menores que $30 \mathrm{ng} / \mathrm{mL}$.

As covariáveis do estudo foram: sexo, faixa etária, etnia, renda mensal, atividade física, tabagismo, doenças autorrelatadas, índice de massa corporal (IMC), circunferência da cintura, exposição solar, uso de protetor solar, horário de exposição solar, parte do corpo exposta ao sol, duração da exposição ao sol, frequência de exposição ao sol e tipos de pele.

O IMC consiste na medida do peso corporal (em $\mathrm{kg}$ ) dividido pela estatura (em metros) ele- vada ao quadrado. A aferição do peso foi realizada com o indivíduo posicionado em pé sobre balança eletrônica, com capacidade para 150 quilos e precisão de 50 gramas. A estatura foi mensurada por meio de estadiômetro profissional portátil, com precisão de $0,1 \mathrm{~cm}$, onde o indivíduo era posicionado em pé, com os calcanhares unidos na base de apoio. O IMC dos indivíduos foi classificado de acordo com os pontos de corte para idosos recomendados pelo Ministério da Saúde" até $22 \mathrm{Kg} / \mathrm{m}^{2}$, baixo peso; entre 22 e $26,99 \mathrm{Kg} / \mathrm{m}^{2}$, eutrófico; $27 \mathrm{Kg} / \mathrm{m}^{2}$ ou mais, sobrepeso.

A circunferência da cintura foi mensurada com fita métrica não-extensível, posicionada no ponto médio entre a crista ilíaca e a última costela, sendo classificados com risco para doenças cardiovasculares (DCV) os idosos com essa medida maior ou igual que $102 \mathrm{~cm}$ para homens e $88 \mathrm{~cm}$ para mulheres ${ }^{10}$.

Em relação à atividade física, o indivíduo foi considerado ativo quando relatava realizar pelo menos 150 minutos de atividade física aeróbica de intensidade moderada ou 75 minutos de intensidade vigorosa ao longo da semana ${ }^{11}$.

Para a avaliação de tabagismo, os indivíduos foram questionados quanto ao hábito de fumar atualmente (caracterizado como "sim"), nunca ter fumado (caracterizado como "não") ou ser ex-fumante.

O tipo de pele foi avaliado por meio da escala de Fitzpatrick ${ }^{12}$, que avalia os fototipos da pele com graduação de 1 a 6 , sendo tipo 1 a pele mais clara, que queima com facilidade e não bronzeia, e tipo 6 a pele mais escura, que não queima e sempre bronzeia à exposição solar (indivíduo apontava em uma figura impressa qual considerava a cor mais próxima de sua pele).

\section{Análise estatística}

Para a análise estatística das variáveis do estudo foram estimadas distribuições de frequências relativas, média e desvio-padrão para as variáveis contínuas e, para as variáveis categóricas, foram estimadas proporções. As diferenças entre os grupos foram estimadas utilizando-se o teste de Mann-Whitney após a verificação da normalidade das distribuições e teste $\mathrm{x}^{2}$ para análise das diferenças entre as variáveis categóricas. As variáveis que apresentaram valor de $\mathrm{p}<0,20$ na associação simples foram selecionadas para o cálculo de razões de chances brutas e ajustadas para idade, com modelos diferentes para características gerais, físicas e de saúde e hábitos de exposição solar, mas permaneceram nas tabelas as variáveis 
com valor de $\mathrm{p}<0,05$. O nível crítico utilizado foi $\mathrm{p}<0,05$. As análises foram realizadas utilizando o software Stata, versão 14.

\section{Resultados}

Os 533 participantes incluídos no estudo tinham idade média de 69,6 $\pm 6,7$ anos, sendo o sexo feminino predominante. A maioria dos idosos declarou ser casado $(60,4 \%)$, branco $(54,4 \%)$, ter menos de oito anos de estudo $(71,8 \%)$ e renda igual ou maior a dois salários-mínimos (70,5\%). Ao analisar os indicadores de estado nutricional, encontramos IMC médio de $28,9 \pm 5,4 \mathrm{~kg} / \mathrm{m}^{2}$, sendo a maioria dos idosos classificados com sobrepeso (60,4\%) e com risco para DCV segundo avaliação da circunferência da cintura $(58,7 \%)$. Em relação aos comportamentos relacionados à saúde, $58,7 \%$ nunca fumaram e $64,4 \%$ eram inativos (Tabela 1).

A concentração sérica média de $25 \mathrm{OH} \mathrm{D}$ foi $28,3 \pm 9,2 \mathrm{ng} / \mathrm{mL}$, e a prevalência de insuficiência foi de $64,5 \%$, sendo maior nas mulheres e nos idosos que se declaram de etnia preta/parda/ indígena/amarela/não declarada, categorizada como "outros" (Tabela 1). Não houve diferença com relação a faixa etária, estado civil, renda, escolaridade, aqueles com trabalho remunerado e chefe de família. Na Tabela 1 também se observa maiores prevalências de insuficiência de vitamina $\mathrm{D}$ entre indivíduos classificados com baixo peso e aqueles que apresentavam medida de circunferência da cintura acima dos limites de risco para desenvolvimento de DCV.

Quanto aos dados de saúde dos idosos, a grande maioria relatou diagnóstico médico de três ou mais doenças, sendo as de maior prevalência hipertensão $(61,9 \%)$, problemas relacionados à coluna $(50,5 \%)$, reumatismo $(43,1 \%)$, diabetes (27,6\%), depressão (27,6\%) e DCV (23,6\%). Não foram encontradas diferenças significativas entre a associação dessas doenças e insuficiência de vitamina, assim como ao agrupar os indivíduos pelo número de doenças pré-existentes.

$\mathrm{Na}$ avaliação da regressão logística ajustada para sexo e idade das características gerais, físicas e de saúde dos indivíduos, observou-se que aqueles com etnia "outros", baixo peso e circunferência de cintura elevada apresentaram maior chance de ter insuficiência de vitamina D (Tabela 2).

Os dados da Tabela 3 mostram que a maioria dos idosos declarou se expor ao sol durante deslocamentos do dia a dia (como ir ao mercado, farmácia etc.) e não ter o hábito de usar protetor solar, expor-se menos de três vezes durante a semana, durante menos de 15 minutos em média e entre o horário das 9 às 15 horas, sendo as partes mais expostas mãos, braços e rosto. Vale ressaltar que o idoso poderia relatar mais de um hábito de exposição, horário e partes do corpo expostas. Segundo a escala de Fitzpatrick, o fototipo de pele predominante foi o tipo 4 , seguido pelos tipos 2 e 3 .

A proporção de suficiência de vitamina $\mathrm{D}$ foi maior entre os participantes que declararam ter hábito de exposição solar durante atividades de lazer e atividades físicas ( $\mathrm{p}=0,018 \mathrm{e} \mathrm{p}=0,010$, respectivamente). Também houve diferença entre os idosos que relataram exposição entre $9 \mathrm{~h}$ e $15 \mathrm{~h}(\mathrm{p}=0,032)$ e com mãos e braços expostos ( $\mathrm{p}$ $=0,046)$ (Tabela 3 ).

$\mathrm{Na}$ avaliação da regressão logística ajustada para sexo e idade dos hábitos de exposição solar dos indivíduos, observou-se associação negativa entre insuficiência de vitamina $\mathrm{D}$ e indivíduos que relataram hábito de exposição durante atividades de lazer, deslocamentos do dia a dia e prática de atividades físicas, exposição entre $9 \mathrm{e}$ 15 horas e mãos, braços e pernas como áreas expostas (Tabela 4).

\section{Discussão}

Foi encontrada alta prevalência de insuficiência de vitamina $D$ entre os idosos avaliados $(64,5 \%)$, sendo que o sexo, a etnia, a composição corporal e alguns hábitos de exposição solar foram associados a essa insuficiência.

Quanto à elevada prevalência de insuficiência de vitamina $\mathrm{D}$, índices semelhantes foram verificados em pesquisas realizadas com o mesmo perfil de população no país ${ }^{4,13,14}$, enquanto um estudo realizado em Ribeirão Preto-SP ${ }^{15}$, com 81 idosos, encontrou uma prevalência maior (76,5\%) de insuficiência, com níveis séricos de $25 \mathrm{OH}$ D de $24,63 \pm 7,89 \mathrm{ng} / \mathrm{mL}$.

O Brasil está em uma localização geográfica com boa disponibilidade de raios ultravioleta (UVB) durante o ano todo, o que possibilita a exposição dos indivíduos à luz solar e à síntese cutânea de vitamina $\mathrm{D}$ em concentrações adequadas na maioria das estações do ano. No entanto, estudos têm reportado altas prevalências de deficiência e insuficiência dessa vitamina na população. Esse paradoxo pode ser explicado, em parte, pela preocupação em adotar ações de prevenção dos riscos de exposição a altos níveis de incidência solar, independentemente da con- 
Tabela 1. Características gerais, físicas e de saúde dos indivíduos com suficiência e insuficiência de vitamina D. Limeira, Piracicaba e Campinas, 2018-2019.

\begin{tabular}{|c|c|c|c|c|c|c|c|}
\hline \multirow{4}{*}{ Variável } & \multicolumn{2}{|c|}{ Total } & \multirow{2}{*}{\multicolumn{2}{|c|}{$\begin{array}{c}\text { Suficiência } \\
\text { vitamina } D\end{array}$}} & \multirow{2}{*}{\multicolumn{2}{|c|}{$\begin{array}{c}\text { Insuficiência } \\
\text { vitamina } D\end{array}$}} & \multirow{4}{*}{ Valor $\mathbf{p}$} \\
\hline & \multirow{2}{*}{\multicolumn{2}{|c|}{$\mathrm{n}=533$}} & & & & & \\
\hline & & & \multicolumn{2}{|c|}{$\begin{array}{l}\mathbf{n}=189 \\
(35,5 \%)\end{array}$} & \multicolumn{2}{|c|}{$\begin{array}{l}\mathrm{n}=344 \\
(64,5 \%)\end{array}$} & \\
\hline & $\mathbf{n}$ & $\%$ & $\mathbf{n}$ & $\%$ & $\mathbf{n}$ & $\%$ & \\
\hline Sexo & & & & & & & 0,000 \\
\hline Mulheres & 353 & 66,2 & 99 & 52,4 & 254 & 73,8 & \\
\hline Homens & 180 & 33,8 & 90 & 47,6 & 90 & 26,2 & \\
\hline Faixa etária & & & & & & & 0,218 \\
\hline 60-74 anos & 419 & 78,6 & 143 & 75,7 & 276 & 80,2 & \\
\hline 75 anos ou mais & 114 & 21,4 & 46 & 24,3 & 68 & 19,8 & \\
\hline Etnia & & & & & & & 0,026 \\
\hline Brancos & 284 & 53,3 & 113 & 59,8 & 171 & 49,7 & \\
\hline $\begin{array}{l}\text { Outros - pretos, pardos, indígenas, amarelos ou } \\
\text { não declarados }\end{array}$ & 249 & 46,7 & 76 & 40,2 & 173 & 50,3 & \\
\hline Renda mensal ${ }^{\star}$ & & & & & & & 0,059 \\
\hline$<2$ salários-mínimos & 108 & 20,3 & 29 & 15,3 & 79 & 23,0 & \\
\hline$\geq 2$ salários-mínimos & 376 & 70,5 & 138 & 73,1 & 238 & 69,2 & \\
\hline Não declarado & 49 & 9,2 & 22 & 11,6 & 27 & 7,8 & \\
\hline IMC & & & & & & & 0,004 \\
\hline Baixo peso & 44 & 8,3 & 9 & 4,8 & 35 & 10,2 & \\
\hline Eutrofia & 167 & 31,3 & 74 & 39,1 & 93 & 27,0 & \\
\hline Sobrepeso & 322 & 60,4 & 106 & 56,1 & 216 & 62,8 & \\
\hline Circunferência cintura & & & & & & & 0,001 \\
\hline Com risco & 313 & 58,7 & 93 & 49,2 & 220 & 64,0 & \\
\hline Sem risco & 220 & 41,3 & 96 & 50,8 & 124 & 36,0 & \\
\hline Atividade física & & & & & & & 0,225 \\
\hline Ativos & 230 & 43,2 & 91 & 48,1 & 139 & 40,4 & \\
\hline Inativos & 303 & 56,8 & 98 & 51,8 & 205 & 59,6 & \\
\hline Tabagismo & & & & & & & 0,749 \\
\hline Sim & 39 & 7,3 & 14 & 7,4 & 25 & 7,3 & \\
\hline Não & 313 & 58,7 & 107 & 56,6 & 206 & 59,9 & \\
\hline Ex-fumante & 181 & 34,0 & 68 & 36,0 & 113 & 32,8 & \\
\hline
\end{tabular}

n: número amostra; \%: proporção a partir do n; valor p: teste do qui-quadrado de Pearson; * salário-mínimo considerado vigente no momento da entrevista (2018 - R\$954,00; 2019 - R\$998,00); IMC: índice de massa corporal.

Fonte: Autoras.

centração de irradiação solar, cuidado que leva a população a se expor menos ao sol ou a utilizar barreiras físicas/químicas (roupas, chapéus, filtro solar), aumentando assim a prevalência de níveis inadequados de vitamina $\mathrm{D}^{16}$.

Especificamente em relação ao sexo, as mulheres apresentaram menores médias e maior prevalência de insuficiência (OR ajustado 2,54; $\mathrm{p}$ $<0,001)$. Estudo que avaliou 359 idosos em Teresina-PI ${ }^{7}$ constatou média de níveis séricos ainda menores $(22,51 \pm 8.03 \mathrm{ng} / \mathrm{mL})$, sendo também mais baixos entre as mulheres. Vários estudos ${ }^{17-19}$ mostram que as mulheres são mais propensas a níveis mais baixos de $25 \mathrm{OH} \mathrm{D}$ do que os homens. Isso pode ocorrer especialmente devido ao tipo de roupas e ao comportamento de proteção solar das mulheres, o que torna a síntese cutânea de vitamina $\mathrm{D}$ menos eficiente ${ }^{20}$.

Em relação a cor da pele, já é bem evidenciado na literatura a eficiência reduzida na síntese de vitamina $\mathrm{D}$ em pessoas com cor de pele escura, devido à maior quantidade de melanina, que 
Tabela 2. Razões de chances (OR) brutas e ajustadas de insuficiência de vitamina $\mathrm{D}$ em relação às características sociodemográficas e de saúde de idosos. Limeira, Piracicaba e Campinas, 2020.

\begin{tabular}{|c|c|c|c|c|}
\hline Variável analisada & OR bruto & Valor $\mathbf{p}$ & $\begin{array}{c}\text { OR } \\
\text { ajustado }^{+}\end{array}$ & Valor $\mathbf{p}$ \\
\hline \multicolumn{5}{|l|}{ Sexo } \\
\hline Masculino & 1,00 & & 1,00 & \\
\hline Feminino & 2,58 & $<0,001$ & 2,54 & $<0,001$ \\
\hline \multicolumn{5}{|l|}{ Etnia } \\
\hline Brancos & 1,00 & & 1,00 & \\
\hline $\begin{array}{l}\text { Outros - pretos, pardos, indígenas, amarelos ou não } \\
\text { declarados }\end{array}$ & 1,48 & 0,031 & 1,46 & 0,043 \\
\hline \multicolumn{5}{|l|}{ Renda mensal ${ }^{\star}$} \\
\hline$\geq 2$ salários-mínimos & 1,00 & & 1,00 & \\
\hline$<2$ salários-mínimos & 1,58 & 0,057 & 1,29 & 0,303 \\
\hline Não declarado & 0,68 & 0,226 & 0,67 & 0,219 \\
\hline \multicolumn{5}{|l|}{ IMC } \\
\hline Eutrófico & 1,00 & & 1,00 & \\
\hline Baixo peso & 3,09 & 0,005 & 2,56 & 0,023 \\
\hline Sobrepeso & 1,60 & 0,016 & 1,41 & 0,085 \\
\hline \multicolumn{5}{|l|}{ Circunferência cintura } \\
\hline Sem risco & 1,00 & & 1,00 & \\
\hline Com risco & 1,83 & 0,001 & 1,57 & 0,018 \\
\hline
\end{tabular}

OR: odds ratio; ${ }^{+}$Ajuste realizado pelas variáveis sexo e idade; ${ }^{*}$ salário-mínimo considerado vigente no momento da entrevista

(2018 - R\$954,00; 2019 - R\$998,00); IMC: índice de massa corporal.

Fonte: Autoras.

atua como um protetor solar natural ${ }^{5}$. Isso explica a maior prevalência de insuficiência de vitamina $\mathrm{D}$ encontrada entre indivíduos que declaram ser de etnias de cor de pele mais pigmentadas.

A associação entre excesso de peso e insuficiência de vitamina D é bem evidenciada na literatura ${ }^{21-27}$. Isso pode ser explicado por fatores biológicos, uma vez que a obesidade diminui a biodisponibilidade de vitamina $\mathrm{D}$ obtida por síntese cutânea, devido à tendência de a vitamina se depositar no tecido adiposo, alterando a contrarregulação de PTH e a síntese hepática de 25 $\mathrm{OH} \mathrm{D}$, além de envolver fatores de estilo de vida, como menor ingestão de vitamina $\mathrm{D}$ na $\operatorname{dieta}^{28}$. Apesar do presente estudo não encontrar associação para sobrepeso pela classificação do IMC, após análise de regressão ajustada, verifica-se essa associação para circunferência da cintura aumentada (OR ajustado 1,57; $\mathrm{p}=0,018$ ). O estudo de Snijder e colaboradores ${ }^{21}$ realizado em Amsterdam com 453 idosos encontrou associação entre baixos níveis de $25 \mathrm{OH}$ D e circunferência da cintura elevada. Essa característica se mostra um importante fator, uma vez que a obesidade abdominal parece tornar o indivíduo mais propenso a menores níveis de $25 \mathrm{OH} \mathrm{D}^{29,30}$.
Por outro lado, encontramos forte associação de insuficiência de vitamina $\mathrm{D}$ e baixo peso (OR ajustado 2,56; $\mathrm{p}=0,023)$. Estudo realizado por $\mathrm{Cabral}^{31}$, que avaliou idosos em Porto Alegre/RS, também encontrou níveis deficientes de $25 \mathrm{OH} \mathrm{D}$ com maior prevalência entre os longevos magros ou desnutridos, sugerindo que níveis deficientes da vitamina D estão associados a risco nutricional e desnutrição.

Diversos estudos mostram maiores níveis séricos de $25 \mathrm{OH} \mathrm{D}$ entre indivíduos que praticam atividade física ${ }^{21,28,32,33}$. Essa característica ainda é acentuada quando essa prática é realizada ao ar livre ${ }^{19}$. No presente estudo, não encontramos diferença significativa entre os grupos para indivíduos ativos. No entanto, indivíduos que relataram hábito de exposição solar durante prática de atividades físicas mostraram menor prevalência de insuficiência de vitamina D.

Entre as doenças relatadas, mesmo sendo bem evidenciado na literatura a relação entre hipovitaminose $\mathrm{D}$ e diversas doenças como diabetes, doenças cardiovasculares, hipertensão arterial, neoplasias, osteoporose, depressão, entre outras $^{34}$, não encontramos associação entre essas doenças e insuficiência de vitamina D. Também 
Tabela 3. Hábitos de exposição solar dos indivíduos com suficiência e insuficiência de vitamina D. Limeira, Piracicaba e Campinas, 2018-2019.

\begin{tabular}{|c|c|c|c|c|c|c|c|}
\hline \multirow{4}{*}{ Variável } & \multirow{2}{*}{\multicolumn{2}{|c|}{ Total }} & \multirow{2}{*}{\multicolumn{2}{|c|}{$\begin{array}{c}\text { Suficiência } \\
\text { vitamina } D\end{array}$}} & \multirow{2}{*}{\multicolumn{2}{|c|}{$\begin{array}{c}\text { Insuficiência } \\
\text { vitamina } D\end{array}$}} & \multirow{4}{*}{$\begin{array}{c}\text { Valor } \\
\text { p }\end{array}$} \\
\hline & & & & & & & \\
\hline & \multicolumn{2}{|c|}{$\mathrm{n}=533$} & \multicolumn{2}{|c|}{$n=189(35,5 \%)$} & \multicolumn{2}{|c|}{$\mathrm{n}=344(64,5 \%)$} & \\
\hline & $\mathbf{n}$ & $\%$ & $\mathbf{n}$ & $\%$ & $\mathbf{n}$ & $\%$ & \\
\hline \multicolumn{8}{|l|}{ Exposição solar } \\
\hline Durante atividades de lazer & 137 & 25,7 & 60 & 31,7 & 77 & 22,4 & 0,018 \\
\hline Durante deslocamentos dia a dia & 408 & 76,5 & 153 & 81,0 & 255 & 74,1 & 0,075 \\
\hline Durante atividades físicas & 190 & 35,6 & 81 & 42,9 & 109 & 31,7 & 0,010 \\
\hline Com finalidade de saúde & 83 & 15,6 & 35 & 18,5 & 48 & 14,0 & 0,164 \\
\hline Uso de protetor solar & & & & & & & 0,207 \\
\hline Sim & 188 & 35,3 & 60 & 31,7 & 128 & 37,2 & \\
\hline Não & 345 & 64,7 & 129 & 68,3 & 216 & 62,8 & \\
\hline \multicolumn{8}{|l|}{ Horário } \\
\hline Antes das 9h & 256 & 48,0 & 85 & 45,0 & 171 & 49,7 & 0,295 \\
\hline Entre $9 \mathrm{~h} \mathrm{e} 15 \mathrm{~h}$ & 274 & 51,4 & 109 & 57,7 & 165 & 48,0 & 0,032 \\
\hline Após 15h & 95 & 17,8 & 33 & 17,5 & 62 & 18,0 & 0,871 \\
\hline \multicolumn{8}{|l|}{ Partes do corpo expostas } \\
\hline Rosto & 418 & 78,4 & 149 & 78,8 & 269 & 78,2 & 0,864 \\
\hline Mãos e braços & 474 & 88,9 & 175 & 92,6 & 299 & 86,9 & 0,046 \\
\hline Pernas & 272 & 51,0 & 107 & 56,6 & 165 & 48,0 & 0,056 \\
\hline Duração média & & & & & & & 0,115 \\
\hline$<15$ minutos & 435 & 81,6 & 161 & 85,2 & 274 & 79,7 & \\
\hline$\geq 15$ minutos & 98 & 18,4 & 28 & 14,8 & 70 & 20,3 & \\
\hline Frequência & & & & & & & 0,718 \\
\hline$<3$ dias por semana & 350 & 65,7 & 126 & 66,7 & 224 & 65,1 & \\
\hline$\geq 3$ dias por semana & 183 & 34,3 & 63 & 33,3 & 120 & 34,9 & \\
\hline Tipo de pele (escala Fitzpatrick) & & & & & & & 0,542 \\
\hline Tipo 1 & 70 & 13,1 & 23 & 12,2 & 47 & 13,7 & \\
\hline Tipo 2 & 142 & 26,6 & 52 & 27,5 & 90 & 26,2 & \\
\hline Tipo 3 & 107 & 20,1 & 43 & 22,7 & 64 & 18,6 & \\
\hline Tipo 4 & 144 & 27,0 & 48 & 25,4 & 96 & 27,9 & \\
\hline Tipo 5 & 39 & 7,3 & 10 & 5,3 & 29 & 8,4 & \\
\hline Tipo 6 & 20 & 3,8 & 7 & 3,7 & 13 & 3,8 & \\
\hline Não declarado & 11 & 2,1 & 6 & 3,2 & 5 & 1,4 & \\
\hline
\end{tabular}

Fonte: Autoras.

não houve diferenças significativas com relação ao número de doenças relatadas.

Presume-se que um filtro solar com fator de proteção solar (FPS) 30 aplicado corretamente reduz de $95 \%$ a $99 \%$ a capacidade da pele em produzir vitamina $\mathrm{D}^{35}$. Apesar disso, não encontramos diferença significativa para insuficiência de vitamina $\mathrm{D}$ entre indivíduos que declaram usar protetor solar ou não. Resultado semelhante foi relatado por Maeda e colaboradores ${ }^{19}$ com o uso ou não de protetor solar. Em contrapartida, no estudo realizado por $\mathrm{Cabral}^{31}$, o uso de filtro solar foi significativamente associado a níveis adequados de $25 \mathrm{OH} \mathrm{D}$, fato que, segundo o autor, pode ser explicado por possível aplicação do filtro solar em quantidade insuficiente ou de forma não homogênea.

A eficiência da exposição solar na síntese de vitamina $\mathrm{D}$ depende não só do uso de protetor solar, mas de fatores como latitude, estação do 
Tabela 4. Razões de chances (OR) brutas e ajustadas de insuficiência de vitamina D em relação aos hábitos de exposição solar de idosos. Limeira, Piracicaba e Campinas, 2020.

\begin{tabular}{|c|c|c|c|c|}
\hline Variável analisada & OR bruto & Valor $\mathbf{p}$ & $\begin{array}{c}\text { OR } \\
\text { ajustado }^{+}\end{array}$ & Valor $\mathbf{p}$ \\
\hline \multicolumn{5}{|l|}{ Exposição solar } \\
\hline \multicolumn{5}{|l|}{ Durante atividades de lazer } \\
\hline Não & 1,0 & & 1,0 & \\
\hline $\operatorname{Sim}$ & 0,62 & 0,018 & 0,63 & 0,030 \\
\hline \multicolumn{5}{|l|}{ Durante deslocamentos dia a dia } \\
\hline Não & 1,0 & & 1,0 & \\
\hline Sim & 0,67 & 0,076 & 0,57 & 0,019 \\
\hline \multicolumn{5}{|l|}{ Durante atividades físicas } \\
\hline Não & 1,0 & & 1,0 & \\
\hline $\operatorname{Sim}$ & 0,61 & 0,010 & 0,67 & 0,040 \\
\hline \multicolumn{5}{|l|}{ Com finalidade de saúde } \\
\hline Não & 1,0 & & 1,0 & \\
\hline Sim & 0,71 & 0,166 & 0,71 & 0,172 \\
\hline \multicolumn{5}{|l|}{ Horário } \\
\hline \multicolumn{5}{|l|}{ Antes das $9 \mathrm{~h}$} \\
\hline Não & 1,0 & & 1,0 & \\
\hline Sim & 1,20 & 0,219 & 1,20 & 0,326 \\
\hline \multicolumn{5}{|l|}{ Entre $9 \mathrm{~h}$ e $15 \mathrm{~h}$} \\
\hline Não & 1,0 & & 1,0 & \\
\hline Sim & 0,67 & 0,032 & 0,68 & 0,042 \\
\hline \multicolumn{5}{|l|}{ Após $15 \mathrm{~h}$} \\
\hline Não & 1,0 & & 1,0 & \\
\hline Sim & 1,03 & 0,871 & 1,08 & 0,744 \\
\hline \multicolumn{5}{|l|}{ Partes do corpo expostas } \\
\hline \multicolumn{5}{|l|}{ Rosto } \\
\hline Não & 1,0 & & 1,0 & \\
\hline $\operatorname{Sim}$ & 0,96 & 0,864 & 0,83 & 0,442 \\
\hline \multicolumn{5}{|l|}{ Mãos e Braços } \\
\hline Não & 1,0 & & 1,0 & \\
\hline Sim & 0,53 & 0,049 & 0,46 & 0,019 \\
\hline \multicolumn{5}{|l|}{ Pernas } \\
\hline Não & 1,0 & & 1,0 & \\
\hline Sim & 0,70 & 0,056 & 0,66 & 0,031 \\
\hline
\end{tabular}

OR: odds ratio; ${ }^{+}$ajuste realizado pelas variáveis sexo e idade.

Fonte: Autoras.

ano, poluição do ar, pigmentação da pele e ida$\mathrm{de}^{36}$. Para adultos brancos, a recomendação de exposição ao sol de braços e pernas (que representam $25 \%$ da superfície corporal, em função do uso de shorts e camisas ou blusas de mangas curtas) por cerca de 5 a 15 minutos, entre $9 \mathrm{~h}$ as e $15 \mathrm{~h}$, três vezes na semana (dependendo, claro, de latitude, estação do ano e pigmentação da pele) seria suficiente para a produção de vitamina D necessária. Para idosos, porém, deve-se considerar que a capacidade de síntese cutânea é bastan- te reduzida, cerca de $25 \%$ da capacidade de uma pessoa de 20 anos $^{37}$.

Apesar de a capacidade de fotoprodução de vitamina $\mathrm{D}$ em idosos ser reduzida, Brouwer -Brolsma e colaboradores ${ }^{36}$ observaram associações significativas entre exposição solar habitual e status de $25 \mathrm{OH}$ D nessa população. Dados do estudo empreendido por Maeda e colaboradores ${ }^{19}$ sugerem que a quantidade de radiação UVB na latitude da cidade de São Paulo/SP é suficiente para uma produção adequada de vitamina $\mathrm{D}$, 
mesmo para a população idosa, desde que haja exposição mínima necessária. Porém, vale ressaltar que a recomendação de exposição solar não é suficiente para corrigir a insuficiência ou deficiência de vitamina $\mathrm{D}^{38}$. Os dados obtidos no presente estudo relacionados à exposição solar vão ao encontro desses achados da literatura. Observou-se menores proporções de insuficiência de vitamina $\mathrm{D}$ entre os indivíduos que relataram exposição solar durante atividades de lazer e prática de atividades físicas ( $\mathrm{p}=0,018$ e $\mathrm{p}=0,010$, respectivamente), e entre as $9 \mathrm{~h}$ e $15 \mathrm{~h}$, período de pico de radiação solar $(\mathrm{p}=0,032)$.

O fato de utilizarmos apenas o relato do idoso como parâmetro de avaliação da exposição solar habitual pode ser considerado um fator limitante de nosso estudo, uma vez que essa informação está sujeita a sub ou superestimação. Além disso, não avaliamos os dados separadamente por estação do ano. Porém, como não houve coleta de dados durante o inverno, e considerando que na latitude em questão a incidência de raios UVB durante a primavera, o verão e o outono são similares, julgamos os achados de relevância. Outro ponto que consideramos um fator limitante do estudo é o fato de os idosos serem voluntários, e muitos já advindos de grupos de saúde da rede de atenção básica dos municípios, inclusive grupos de atividades físicas ao ar livre, trazendo o viés do “participante/voluntário saudável”, fato que pode ter influenciado em parte nos resultados. No entanto, esta é uma limitação comum em estudos populacionais em que os participantes são convidados em entrevistas não-domiciliares, já que os voluntários normalmente são pessoas com maior interesse em assuntos relacionados à saúde.

Destaca-se como um dos pontos fortes do presente estudo o fato de trabalhar com uma amostra de população idosa assistida por serviços da atenção primária à saúde das cidades de Limeira, Piracicaba e Campinas, além de investigar em um mesmo estudo vários fatores que podem estar associados à deficiência de vitamina $\mathrm{D}$ nessa faixa etária tão vulnerável.

Os resultados obtidos mostram a relevância de fatores como sexo, etnia, composição corporal, atividade física e hábitos de exposição solar na prevalência de níveis inadequados de vitamina $\mathrm{D}$ em idosos, e a importância de considerá-los como preditivos da insuficiência dessa vitamina nessa população, sobretudo como ferramenta da atenção básica. A avaliação desses fatores é uma medida simples, de baixo custo e não invasiva, que pode contribuir para a identificação precoce da insuficiência de vitamina $\mathrm{D}$, possibilitando ações e estratégias de prevenção e controle dessa condição que vem se mostrando cada vez mais prevalente no Brasil e no mundo.

\section{Colaboradores}

PMD Rolizola trabalhou na coleta de dados, análise de dados e redação do manuscrito. CN Freiria contribuiu na coleta de dados, preparação do banco de dados, análise de dados e revisão do manuscrito. GM Silva atuou na coleta de dados, preparação do banco de dados e revisão do manuscrito. TRP Brito e FSA Borim trabalharam na coordenação da pesquisa e na revisão da análise e do manuscrito. LP Corona contribuiu na concepção do estudo, no plano de análises, na coordenação da pesquisa e na revisão da redação do manuscrito. Todos os autores leram e aprovaram o manuscrito final. 


\section{Agradecimentos}

Ao Conselho Nacional de Pesquisa e Desenvolvimento $(\mathrm{CNPq})$, à Pró-Reitoria de Pesquisa da Unicamp e à Coordenação de Aperfeiçoamento de Pessoal de Nível Superior (CAPES), que financiaram a pesquisa (processos: CNPq 408262/20076, FAEPEX 2854/18 e 3150/19, CAPES código de financiamento 001).

\section{Referências}

1. Wimalawansa SJ. Non-musculoskeletal benefits of vitamin D. J Steroid Biochem Mol Biol 2018; 175:60-81.

2. Mendes MM, Hart KH, Botelho PB, Lanham-New SA. Vitamin D status in the tropics: Is sunlight exposure the main determinant? Nutr Bull 2018; 43(4):428-434.

3. Cabral MA, Borges CN, Maia JMC, Aires CAM, Bandeira F. Prevalence of vitamin $\mathrm{D}$ deficiency during the summer and its relationship with sun exposure and skin phototype in elderly men living in the tropics. Clin Interv Aging 2013; 8:1347-1351.

4. Santos CS, Bessa TA, Xavier AJ. Fatores associados à demência em idosos. Cien Saude Colet 2020; 25(2):603-611.

5. Moreira CA, Ferreira CEDS, Madeira M, Silva BCC, Maeda SS, Batista MC, Bandeira F, Borba VZC, Lazaretti-Castro M. Reference values of 25-hydroxyvitamin D revisited: a position statement from the Brazilian Society of Endocrinology and Metabolism (SBEM) and the Brazilian Society of Clinical Pathology/Laboratory Medicine (SBPC). Arch Endocrinol Metab 2020; 64(4):462-478.

6. Oliveira GBVP, Nunes IFOC, Carvalho LR, Figueirêdo RG, Oliveira MCB, Carvalho CMRG. Perfil antropométrico e níveis séricos de vitamina $\mathrm{D}$ de idosos participantes do programa saúde da família de Teresina. Rev Interd Cien Saude 2014; 1(1):48-55.

7. Oliveira GBVP, Silva Júnior FL, Oliveira MCB, Carvalho CMRG. Relação dos índices antropométricos e vitamina $\mathrm{D}$ com o desempenho funcional em idosos TT - Relationship of anthropometric indices and vitamin D with functional performance in elderly. Estud interdiscip Envelhec 2018; 23(1):43-59.

8. Ferreira CES, Maeda SS, Batista MC, Lazaretti-Castro M, Vasconcellos LS, Madeira M, et al. Consensus reference ranges of vitamin $\mathrm{D}[25(\mathrm{OH}) \mathrm{D}]$ from the Brazilian medical societies. Brazilian Society of Clinical Pathology/Laboratory Medicine (SBPC/ML) and Brazilian Society of Endocrinology and Metabolism (SBEM). J Bras Patol Med Lab 2017; 56(6):377-381.

9. Brasil. Ministério da Saude (MS). Orientações para a coleta e análise de dados antropométricos em serviços de saúde: Norma Técnica do Sistema de Vigilância Alimentar e Nutricional - SISVAN. Brasília: MS; 2011.

10. World Heath Organization (WHO). WHO 1995 Physical status. Use and anthropometry. World Health Organization technical report series 1995.

11. World Heath Organization (WHO). Global recommendations on physical activity for health. Geneva: WHO; 2010;

12. Fitzpatrick TB. The Validity and Practicality of Sun -Reactive Skin Types I Through VI. Arch Dermatol 1988; 124(6):869-871.

13. Saraiva GL, Cendoroglo MS, Ramos LR, Araújo LM, Vieira JG, Kunii I, Hayashi LF, Corrêa MP, LazarettiCastro M. Influence of ultraviolet radiation on the production of 25 hydroxyvitamin $\mathrm{D}$ in the elderly population in the city of São Paulo (23 $34^{\prime}$ S), Brazil. Osteoporos Int 2005; 16(12):1649-1654.

14. Saraiva GL, Cendoroglo MS, Ramos LR, Araújo LMQ, Vieira JGH, Maeda SS, Borba VZC, Kunii I, Hayashi LF, Lazaretti-Castro M. Prevalência da deficiência, insuficiência de vitamina D e hiperparatiroidismo secundário em idosos institucionalizados e moradores na comunidade da cidade de São Paulo, Brasil. Arq Bras Endocrinol Metabol 2007; 51(3):437-442. 
15. Cicilini AL, Singling MV, Silva KA, Ramos APP. Níveis séricos de 25-hidroxivitamina $\mathrm{D}$ em idosos com Diabetes mellitus tipo 2 atendidos em um Hospital Universitário de Ribeirão Preto (SP). Rev Ciências da Saúde da Amaz 2019; 1:3-24.

16. Pereira-Santos M, Santos JYG, Carvalho GQ, Santos DB, Oliveira AM. Epidemiology of vitamin D insufficiency and deficiency in a population in a sunny country: Geospatial meta-analysis in Brazil. Crit Rev Food Sci Nutr 2019; 59:2102-2109.

17. Dawson-Hughes B, Harris SS, Dallal GE. Plasma calcidiol, season, and serum parathyroid hormone concentrations in healthy elderly men and women. Am J Clin Nutr 1997; 65(1):67-71.

18. Carnevale V, Modoni S, Pileri M, Di Giorgio A, Chiodini I, Minisola S, Vieth R, Scillitani A. Longitudinal evaluation of vitamin $\mathrm{D}$ status in healthy subjects from southern Italy: Seasonal and gender differences. Osteoporos Int 2001; 12(12):1026-1230.

19. Maeda SS, Kunii IS, Hayashi LF, Lazaretti-Castro M. Increases in summer serum 25-hydroxyvitamin D (25OHD) concentrations in elderly subjects in São Paulo, Brazil vary with age, gender and ethnicity. BMC Endocr Disord 2010; 10:12.

20. Nimitphong H, Holick MF. Vitamin D status and sun exposure in Southeast Asia. Dermatoendocrinol 2013; 5(1):34-37.

21. Snijder MB, van Dam RM, Visser M, Deeg DJ, Dekker JM, Bouter LM, Seidell JC, Lips P. Adiposity in relation to vitamin $\mathrm{D}$ status and parathyroid hormone levels: A population-based study in older men and women. $J$ Clin Endocrinol Metab 2005; 90(7):4119-4123.

22. Scragg R, Camargo CA. Frequency of leisure-time physical activity and serum 25-hydroxyvitamin D levels in the US population: Results from the third national health and nutrition examination survey. $\mathrm{Am} \mathrm{J}$ Epidemiol 2008; 168(6):577-586.

23. Lagunova Z, Porojnicu LC, Lindberg F, Hexeberg S, Moan J. The dependency of vitamin D status on body mass index, gender, age and season. In: Anticancer Research. 2009. p. 3713-20.

24. Jorde R, Sneve M, Emaus N, Figenschau Y, Grimnes G. Cross-sectional and longitudinal relation between serum 25-hydroxyvitamin D and body mass index: The Tromsø study. Eur J Nutr 2010; 49(7):401-407.

25. Yoshimura N, Muraki S, Oka H, Morita M, Yamada H, Tanaka S, Kawaguchi H, Nakamura K, Akune T. Profiles of vitamin D insufficiency and deficiency in Japanese men and women: association with biological, environmental, and nutritional factors and coexisting disorders: the ROAD study. Osteoporos Int 2013; 24(11):2775-2787.

26. Nakamura K, Kitamura K, Takachi R, Saito T, Kobayashi R, Oshiki R, Watanabe Y, Tsugane S, Sasaki A, Yamazaki O. Impact of demographic, environmental, and lifestyle factors on vitamin D sufficiency in 9084 Japanese adults. Bone 2015; 74:10-17.

27. Mansouri M, Abasi R, Nasiri M, Sharifi F, Vesaly S, Sadeghi O, Rahimi N, Sharif NA. Association of vitamin D status with metabolic syndrome and its components: a cross-sectional study in a population of high educated Iranian adults. Diabetes Metab Syndr Clin Res Rev 2018; 12(3):393-398.
28. Jääskeläinen T, Knekt P, Marniemi J, Sares-Jäske L Männistö S, Heliövaara M, Järvinen R. Vitamin D status is associated with sociodemographic factors, lifestyle and metabolic health. Eur J Nutr 2013; 52(2):513525.

29. Gheno FP, Cabral MMS, Schirmer CL, Dallepiane LB, Bos AJG. Diet quality and nutritional status in Southern Brazilian oldest old. Nutr Food Toxicol. 2017;1:2

30. Matchar DB, Chei CL, Yin ZX, Koh V, Chakraborty B, Shi XM, Zeng Y. Vitamin D levels and the risk of cognitive decline in Chinese elderly people: the Chinese Longitudinal Healthy Longevity Survey. J Gerontol A Biol Sci Med Sci 2016; 71(10):1363-1368.

31. Cabral MMS. Vitamina D em longevos, qual o fator mais importante: ingesta alimentar ou exposição solar? [Tese]. Porto Alegre: Pontifícia Universidade Católica do Rio Grande do Sul; 2017.

32. Looker AC. Do body fat and exercise modulate vitamin D status? Nutr Rev 2007; 65(8 Pt. 2):S124-S126.

33. Hintzpeter B, Mensink GBM, Thierfelder W, Müller MJ, Scheidt-Nave C. Vitamin D status and health correlates among German adults. Eur J Clin Nutr 2008; 62(9):1079-1089.

34. Caristia S, Filigheddu N, Barone-Adesi F, Sarro A, Testa T, Magnani C, Aimaretti G, Faggiano F, Marzullo P. Vitamin D as a biomarker of ill health among the over-50s: a systematic review of cohort studies. $\mathrm{Nu}$ trients 2019;11

35. Hossein-Nezhad A, Holick MF. Vitamin D for health: a global perspective. In: Mayo Clinic Proceedings. 2013 p. $720-755$

36. Brouwer-Brolsma EM, Vaes AMM, van der Zwaluw NL, van Wijngaarden JP, Swart KMA, Ham AC, van Dijk SC, Enneman AW, Sohl E, van Schoor NM, van der Velde N, Uitterlinden AG, Lips P, Feskens EJM, Dhonukshe-Rutten RAM, de Groot LCPGM. Relative importance of summer sun exposure, vitamin $\mathrm{D}$ intake, and genes to vitamin D status in Dutch older adults: The B-PROOF study. J Steroid Biochem Mol Biol 2016; 164:168-176.

37. Holick MF. Vitamin D: a D-lightful solution for health. In: Journal of Investigative Medicine. 2011. p. 872880.

38. Lee YM, Kim SA, Lee DH. Can current recommendations on sun exposure sufficiently increase serum vitamin D level?: One-month randomized clinical trial. J Korean Med Sci. 2020; 35:8.

Artigo apresentado em 05/10/2020

Aprovado em 07/01/2021

Versão final apresentada em 09/01/2021

Editores-chefes: Romeu Gomes, Antônio Augusto Moura da Silva 
